\title{
The Linear Equation for 2D Graphs
}

\author{
Rumani Dey
}

Abstract:

What is $\mathbf{y}=\mathbf{m x}+\mathbf{c}$ ?

What is one dimensional?

If a thing like fish/bird while in motion in water/air cannot turn left, right and backward. It can only move forward in a medium or fly in air. When I try to determine its motion, I see it as one dimensional. It is not necessary that the bird/fish is moving in a straight line. It is the sheer concentration of the medium the fish /bird is traversing in. So, the behavior of a one dimensional motion maybe a function of the medium with a concentration/penetration quotient of the medium based on the weight of the fish/bird which penetrates the medium and the effect of gravity on it. We can depict a one dimensional motion as,

One Dimensional motion $=\mathbf{f}($ weight $) * \mathbf{f}($ penetration quotient)*f(Force exerted by the fish/bird while it is penetrating the medium)

What is one dimensional coordinate?

We can say that it is a vector $(V)$ which moves in any direction but not necessarily a straight line . If " $x$ " is a one dimensional coordinate value, it is very difficult to find the value of " $x$ " . If suppose the path traversed by " $x$ " is not a definite symmetrical pattern but an ambiguous pattern like below:

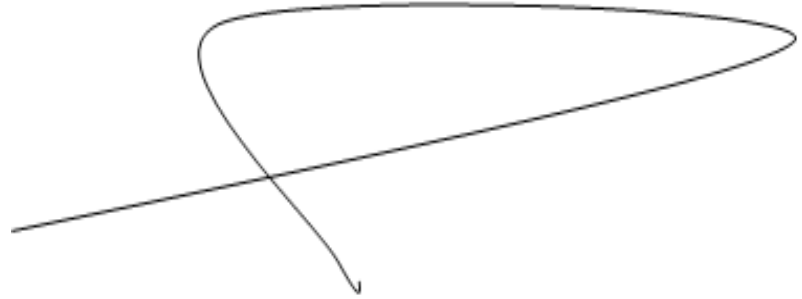

Fig 1
Then we do not have a numerical value for " $x$ ". It is not measurable through a scale. We cannot find the value of " $x$ ". In this case the only way " $x$ " can be measured is through a straight line if " $x$ " is moving in one direction only. A question arises whether time is moving in a circular pattern or in a straight line. We measure it as a distance/displacement and we have deemed such a scale of measurement as one dimensional. But I want to measure the quantity the fish/bird had travelled in one minute. We might get some value by defining the area it had traversed in one minute which is a two dimensional quantity.

\section{INTRODUCTION}

This piece is not a copy of the metrics in the equation $y=m x+c$. It is actually an original attempt to derive the metrics from this equation. I converted this to a paper since I carry pleasure to publish every original effort . The purpose behind this paper is too bring to notice to the school curriculum to not blindly impose some equations and get the metrics values but to derive at the end points of the metrics. Science is not different from Math. To think analytically and solve an already established equation gives pleasure and confidence. This paper is not a work of philosophy yet. It is the simple derivation only. The science behind these metrics is yet curious. But I am not ready to do this right now. Meanwhile any student can go through this paper to gain knowledge and apply their analytical skills to achieve the fun Math brings. I have started from scratch again to brush up my high school knowledge. I know this paper is the beginning of a new project whose end results are yet to be seen.

\section{What is $\mathbf{y}=\mathbf{m x}+\mathbf{c}$ ?}

We know that two dimensional measurements can be expressed as graphs. We have an equation for the linear graph as $\mathrm{y}=\mathrm{mx}+\mathrm{c}$ where $(\mathrm{x}, \mathrm{y})$ are coordinates. By convention if I have the coordinates in a graph as $(0,0)$, $(0,1),(1,0),(1,1)$. If we plot the coordinates we get-

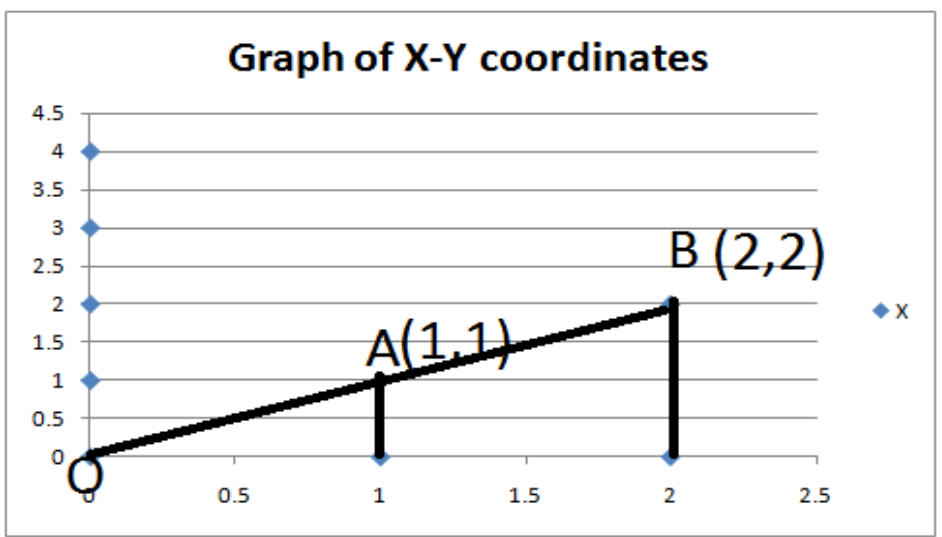

Fig 2 
As per $\mathrm{y}=\mathrm{mx}+\mathrm{c}$,

$(0,0) \rightarrow 0=m \cdot 0+\mathrm{c} \rightarrow \mathrm{c}=0$

$(0,1) \rightarrow 1=m .0+c \rightarrow c=1$

$(1,0) \rightarrow 0=m .1+c \rightarrow c=-m$

$(1,1) \rightarrow 1=m .1+c \rightarrow c=1-m$

So, the constant "c" is changing as per the coordinates.

$\mathrm{OA}$ is $(0,0)$ and $(1,1)$

So, $\mathrm{y}=\mathrm{mx}+\mathrm{c}$ gives $\mathbf{c}=\mathbf{0}$ for $(\mathrm{x}, \mathrm{y})=(0,0)$

$\rightarrow \mathrm{c}=1-\mathrm{m} \rightarrow 0=1-\mathrm{m} \rightarrow \mathrm{m}=1$

So, $\mathrm{y}=\mathrm{x}+0 \rightarrow \mathrm{y}=\mathrm{x}$

$\mathrm{OB}$ is $(0,0)$ and $(2,2)$

So, $\mathrm{y}=\mathrm{mx}+\mathrm{c}$ gives $\mathbf{c}=\mathbf{0}$ for $(\mathrm{x}, \mathrm{y})=(0,0)$

$\rightarrow 2=2 \mathrm{~m}+\mathrm{c} \rightarrow 2=2 \mathrm{~m}+0 \rightarrow \mathrm{m}=1$

Hence $\mathrm{y}=1 \mathrm{x}+0=\mathrm{x}$
$\mathrm{AB}$ is $(1,1)$ and $(2,2)$

$\mathrm{y}=\mathrm{m}+\mathrm{c} \rightarrow 1=\mathrm{m}+\mathrm{c}------(\mathrm{Eq} 1)$

$\mathrm{y}=2 \mathrm{~m}+\mathrm{c} \rightarrow 2=2 \mathrm{~m}+\mathrm{c}---(\mathrm{Eq} 2)$

Solving both the equations we get, $\mathrm{m}=1-\mathrm{c}$ from $\mathrm{Eq} 1$

Putting the values of " $\mathrm{m}$ " in Eq2, we get $2=2(1-\mathrm{c})+\mathrm{c} \rightarrow \mathrm{c}=0$

$m=1-c \rightarrow$ of $m=1-0=1$

So in this graph for all coordinates the value of $m=1$ and $\mathrm{c}=0$ which makes $\mathrm{y}=\mathrm{x}$.

This is the derivation of $y=m x+c$ and proof that its correct. But what metrics or measurements do " $\mathrm{m}$ " and "c" denote. Lets try this too.

Let's take a value wherein " $x$ " is not equal to " $y$ " $\mathrm{y}=\mathrm{mx}+\mathrm{c}$ for $(1,5)$ and $(-1,4)$. Lets see what the graph brings:

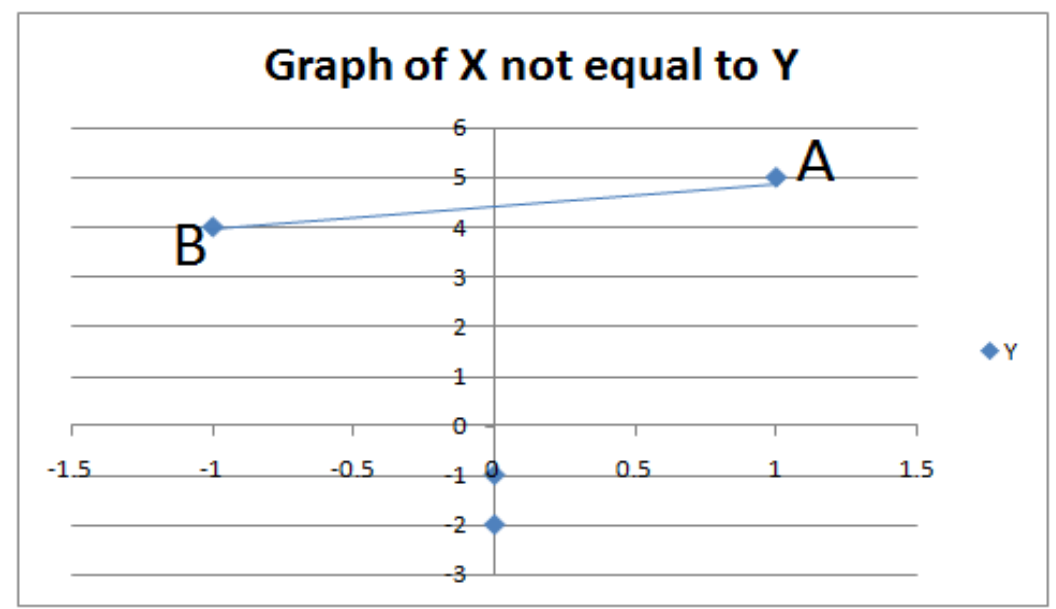

Fig 3

We have joined the points $\mathrm{B}$ and A. Lets solve $\mathrm{y}=\mathrm{mx}+\mathrm{c}$,

$(1,5) \rightarrow 5=m+c---------(E q 1)$

$(-1,4) \rightarrow 4=-m+c-------($ Eq2)

Solving the above equations we get,

Adding Eq1 and Eq2, we get $9=2 \mathrm{c} \rightarrow \mathrm{c}=4.5$

$\mathrm{m}=\mathrm{c}-4$ from $\mathrm{Eq} 2, \rightarrow \mathrm{m}=4.5-4=0.5$

This proves something, $\mathrm{y}=\mathrm{mx}+\mathrm{c}$ does have some significance on the graphs:

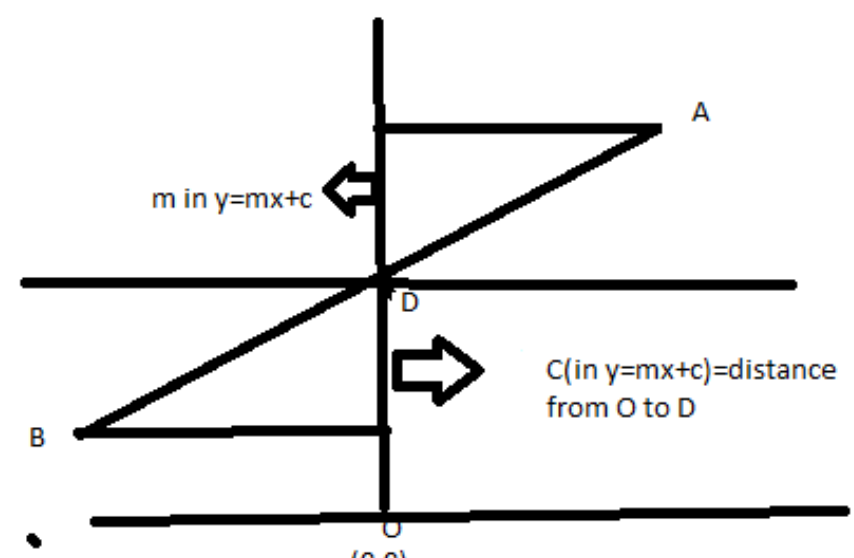

$(0,0)$

Fig 4:- Magnified version of Fig 3 
$\mathrm{m}=$ Perpendicular distance on the $\mathrm{Y}$-axis from from $\mathrm{D}$, if the point $\mathrm{A}$ is intersected to the $\mathrm{Y}$-axis

$\mathrm{c}=$ Intersection point of the $\mathrm{Y}$-axis with $\mathrm{AB}$

Lets see what the length of the linear graph is . By Pythagoras theorem, $\mathrm{DA}=\mathrm{DB}=\sqrt{ }\left\{\left(0.5^{\wedge} 2\right)+\left(1^{\wedge} 2\right)\right\}=0.5$

$\mathrm{AB}=1$

So the equation of $\mathrm{AB}$ is as follows:

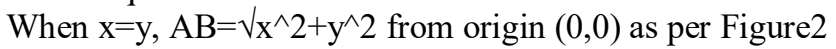

$y=m x+c=($ Distance from the intersection point of the linear graph on the $\mathrm{Y}$ axis till the intersection point of the horizontal line drawn from either $\mathrm{A}$ or $\mathrm{B}$ on the $\mathrm{Y}$ axis )*(Base of the triangle $)+$ Distance of the the origin to the intersection point of the linear graph to the $\mathrm{Y}$ axis

\section{CONCLUSION}

This piece is already derived and established. I am publishing it still as it's an original effort to find the values of $\mathrm{m}, \mathrm{c}$ in the equation for linear graph. Do provide your feedback on it.

\section{REFERENCES}

[1]. Mathematics Text-Book for Class X1 by NCERT publication-Chapter 2 - Relations and functions 